\title{
Erratum
}

\section{The limiting spectral distribution in terms of spectral density}

\author{
[Random Matrices Theory and Applications, Vol. 5, No. 1 (2016), \\ DOI: $10.1142 / \mathrm{S} 2010326316500039]$ \\ Costel Peligrad* and Magda Peligrad ${ }^{\dagger}$ \\ Department of Mathematical Sciences, University of Cincinnati \\ P. O. Box 210025, Cincinnati, OH 45221-0025, USA \\ *peligrc@ucmail.uc.edu \\ †peligrm@ucmail.uc.edu
}

Accepted 30 August 2017

Published 8 September 2017

\begin{abstract}
Keywords: Random matrices; correlated entries; limiting spectral distribution; stationary random fields; spectral density.

Mathematics Subject Classification 2010: 60F15, 60G60, 60G10, 62E20, 42A38
\end{abstract}

There is a misprint in formula (7) of Theorem 10 There is no misprint in its proof. The correct formulation is:

Theorem 1. Let $\left(X_{k, \ell}\right)_{(k, \ell) \in \mathbb{Z}^{2}}$ be a real-valued random field given by (3) with a spectral density $f(x, y)$. Define its scaling $b(x, y)$ by (5) and assume that $\gamma_{k, \ell}=\gamma_{\ell, k}$ for all $(k, \ell) \in \mathbb{Z}^{2}$. Then, the convergence (4) holds, namely $F^{\mathbb{X}_{N}(\omega)} \Rightarrow F$ a.s., where $F$ is a nonrandom limiting distribution function whose Stieltjes transform $S(z)$ is uniquely defined by the relations: for every $z \in \mathbb{C}^{+}$

$$
S(z)=\int_{0}^{1} g(x, z) \mathrm{d} x,
$$

where, for any $x \in[0,1], g(x, z)$ is analytic in $z \in \mathbb{C}^{+}$.

There is $J \subset[0,1]$, with $\lambda(J)=1$ such that, for any $x \in J$ and $z \in \mathbb{C}^{+}, g(x, z)$ satisfies the equation

$$
g(x, z)=-\left(z+\int_{0}^{1} g(y, z) b(x, y) \mathrm{d} y\right)^{-1} .
$$

Moreover, for any $x \in[0,1]$ and $z \in \mathbb{C}^{+}$

$$
\operatorname{Im} g(x, z)>0, \quad|g(x, z)| \leq(\operatorname{Im} z)^{-1} .
$$

